\title{
Human circadian variations
}

\author{
Nicholas W. Gentry, ${ }^{1}$ Liza H. Ashbrook, ${ }^{1,3}$ Ying-Hui Fu, ${ }^{1,2,3,4}$ and Louis J. Ptáček ${ }^{1,2,3,4}$ \\ ${ }^{1}$ Department of Neurology, ${ }^{2}$ Institute for Human Genetics, ${ }^{3}$ Weill Institute for Neurosciences, and ${ }^{4}$ Kavli Institute for Fundamental Neuroscience, UCSF, San Francisco, California, USA.
}

\begin{abstract}
Circadian rhythms, present in most phyla across life, are biological oscillations occurring on a daily cycle. Since the discovery of their molecular foundations in model organisms, many inputs that modify this tightly controlled system in humans have been identified. Polygenic variations and environmental factors influence each person's circadian rhythm, contributing to the trait known as chronotype, which manifests as the degree of morning or evening preference in an individual. Despite normal variation in chronotype, much of society operates on a "one size fits all" schedule that can be difficult to adjust to, especially for certain individuals whose endogenous circadian phase is extremely advanced or delayed. This is a public health concern, as phase misalignment in humans is associated with a number of adverse health outcomes. Additionally, modern technology (such as electric lights and computer, tablet, and phone screens that emit blue light) and lifestyles (such as shift or irregular work schedules) are disrupting circadian consistency in an increasing number of people. Though medical and lifestyle interventions can alleviate some of these issues, growing research on endogenous circadian variability and sensitivity suggests that broader social changes may be necessary to minimize the impact of circadian misalignment on health.
\end{abstract}

\section{Introduction}

Measurement of rest and activity in flies by Konopka and Benzer in 1971 (1) marked the beginning of behavioral genetics as a field, and in 2017, the Nobel Prize in Medicine was awarded to Hall, Young, and Rosbash for their "discoveries of molecular mechanisms controlling the circadian rhythm." Work from many laboratories in model systems and in humans has advanced our understanding of the molecular basis of circadian regulation. It has long been evident that circadian (meaning "around a day") behaviors play a role in much of life. Animals, plants, and even bacteria (2) have demonstrated behavioral patterns that follow a near-24-hour rhythm. We now understand that a molecular system genetically coded into each cell guides these patterns through a tightly controlled network of genes and proteins that oscillate in concentration on a daily cycle. Many, though not all, elements of this system are conserved across phyla; though the first biological clock genes were found in flies $(3,4)$, and elucidated further in other model organisms, many of the core components or their analogs are present and operate following similar principles in humans.

In mammals, the central pacemaker of the circadian system is the suprachiasmatic nucleus (SCN) within the hypothalamus, which synchronizes many features of the sleep-wake cycle. It is here that metabolic, environmental, and genetic inputs are integrated to generate the period (also called $\tau$ ), or the length of the circadian cycle for the entire organism, manifested in numerous behavioral and physiological patterns. Related to the period is phase, or the timing of such expressed rhythms (5). In humans, the phase is often measured using the nadir of body temperature or the onset of evening melatonin in the evening in dim light con-

Authorship note: NWG and LHA contributed equally to this work. Conflict of interest: The authors have declared that no conflict of interest exists. Copyright: (5) 2021, American Society for Clinical Investigation.

Reference information: / Clin Invest. 2021;131(16):e148282.

https://doi.org/10.1172/JCl148282. ditions (dim-light melatonin onset [DLMO]) as reliable reference points for circadian timing (6).

These features of the internal cyclic clock are entrained, or synchronized to the environment, by external cues, known as "zeitgebers" (from German, "time givers"), without which "drift" may occur, leading to gradual desynchronization with solar rhythms. Entrainment to zeitgebers is what helps reestablish circadian time when the biological clock machinery is desynchronized from solar time, such as the jet lag that arises as a result of transmeridian air travel. By far the strongest zeitgeber in mammals is light, though social cues, food intake (7), and exercise (8) play a minor role. Light cues transmit signals to the SCN in the brain that entrain the circadian phase to match the input from the environment. The relationship between exposure to extrinsic drivers such as light and the circadian phase is called the phase angle of entrainment.

An organism's endogenous circadian length is best measured in contexts free of strong zeitgebers such as light. In the absence of time cues, the average human circadian rhythm cycles about once every 24.2 hours $(9,10)$ and can slowly desynchronize from the 24-hour solar day (11). Evidence for this desynchronization comes from desynchronization experiments (12) and individuals with the rare non-24-hour sleep-wake rhythm disorder (13).

\section{Genetic underpinnings of the circadian system}

Investigation in animal models $(14,15)$ and humans $(16)$ has found that circadian rhythms are influenced by genetic variation. An individual human's circadian period length can be significantly shorter or longer than the 24.2-hour average (16-19). The extremes of this normal variation appear to be strongly driven by genetics, along with other features of circadian periodicity in humans; even sensitivity to zeitgebers has been shown to be genetically variable (20). Individual variations in phase, phase angle of entrainment, and period, along with environmental factors and age, help generate what is known as "chronotype," the unique daily activity patterns of each person. In the general population, these strong 


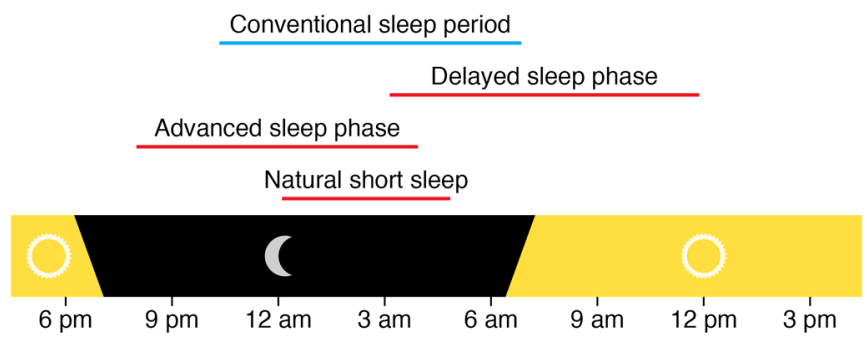

Figure 1. Conventional sleep period timing compared with delayed sleep phase, advanced sleep phase, and natural short sleep. Note that the "conventional sleep period" can be very variable. The timing of "conventional sleep" in this figure (10 pm to $7 \mathrm{am})$ is simply one example close to the population average. Adapted with permission from Neuropsychopharmacology (127).

genetic determinants are presumably the sum of many genetic variations of small effect. Early chronotypes have sleep-wake cycles phase-shifted earlier in the day, resulting in a morning preference, while late chronotypes have an evening preference (21). Environmental factors such as artificial lighting can play a major role in sleep timing as demonstrated by research showing that camping, with a large amount of natural light and absent artificial light exposure, can lead to a marked advance of sleep timing and melatonin offset $(22,23)$. Chronotype can be somewhat reliably and simply determined using validated tools $(17,24)$, but our understanding of the drivers of period and phase is still in nascent stages, as each person's preferred sleep-wake timing and associated circadian period are determined by the complex interplay of an unknown number of genetic and environmental variables (16). As a result, much of our knowledge of how genetics can influence circadian rhythms comes from reverse genetics using animal models or the study of individuals at the most extreme ends of human variation in phase. Single mutations in core circadian clock genes can lead to variations with strong effects on the molecular timekeeping system (25-27).

The core human molecular clock consists of a network of timekeeping genes and their associated proteins, including CLOCK/ BMAL1, CRY, and PER $(28,29)$, in addition to numerous secondary inputs and interacting modulators. Each of these circadian genes is transcribed, translated, and degraded on a consistent daily cycle, and together they constitute a tightly regulated negative-feedback loop: the heterodimeric CLOCK/BMAL1 complex activates transcription of PER and CRY; after translation, these proteins repress the activity of CLOCK/BMAL1 (28). Other molecular inputs, such as retinoic acid receptor-related orphan receptors (RORs) and the REV-ERB proteins NR1D1 and NR1D2 (among others), further modulate circadian phase in response to various signals $(30,31)$. Downstream, the phase of this molecular cycle dictates the transcription of many genes $(32,33)$ - perhaps as many as $43 \%$ of all protein-coding genes (33) - which oscillate in a circadian manner along with their numerous physiological and behavioral consequences. Other forms of regulation, such as posttranslational modifications, found in the case of the casein kinase CK1 $\delta$, further modulate cellular activity as a function of circadian period $(25,34)$. The clock is tightly integrated with metabolism (35), and together these processes influence much of human biology, including hormone balance (36), feeding habits (37) and digestion (38), body temperature, cognition, and many more $(35,39)$.
Extreme circadian traits can arise when one or more components of the biological clock are mutated in such a way as to drastically change phase, phase angle of entrainment, or period length. Even in the presence of zeitgebers, individuals with these traits may experience difficulties staying on a conventional schedule. Depending on the timing, light can either advance or delay the phase of an individual's circadian period, resulting in what is known as the phase-response curve (PRC) of human responsiveness to this zeitgeber (40). Early morning light tends to advance phase, while evening light delays it (41). As light exposure modifies circadian clock phase, in our light-inundated modern world, the dearth of consistency in external light cues may exacerbate any circadian disorder phenotypes (42-44), and possibly increase their prevalence.

\section{Chronotype across the population}

Chronotype varies across the population in a near-Gaussian distribution (45). Sleep timing is known to shift later during puberty, with the most delayed timing around age 20, then slowly shifts earlier over subsequent decades (46). It is hypothesized that throughout life people typically maintain their relative chronotype when compared with others of the same age and in the same location (46). The effect of age on chronotype also appears to affect women less than men, leading to increased prevalence of earlier chronotypes in women at younger ages, but the reverse over the age of 40 (21). Additionally, the amplitude of the circadian rhythm as measured by temperature and transcriptional oscillations is reduced with age in mammals (47-49). The biological mechanism for these phenomena is not understood. Amplitude dampening is correlated with the development of neurodegenerative disorders, such as Alzheimer's disease (50) and Parkinson's disease (51), and is exacerbated by these disorders, indicating that the dampening may be at least partially driven by a decoupling of the SCN's connectivity with other parts of the brain or body (52-54).

\section{Extremes of chronotype and genetics}

At one extreme, those with a preference to go to sleep much earlier and awaken much earlier than others relative to local solar time, called advanced sleep phase (ASP), are known as extreme morning larks or early birds (Figure 1). People with ASP often report being lifelong morning larks. When most extreme, this trait is commonly familial and is termed FASP (55). For example, individuals with FASP can consistently fall asleep by 8:30 pm and awaken spontaneously before 5:30 am, though norms vary by age, longitude, and location. The prevalence of FASP and of ASP in a sleep clinic population is estimated to be at least $0.21 \%$ and $0.33 \%$, respectively (55). Several genes have been identified conferring this trait in an autosomal dominant manner, including PER2, CRY2, CSNK1D (encoding CK1ס), PER3, and TIMELESS (25, 27, 56-58), and carriers of these variants report an extreme early-bird preference. Those at the far late extreme of chronotype distribution, who prefer a much later bedtime and later wake time, are said to have delayed sleep phase (DSP) or to be extreme night owls. For example, sleep onset may be at $3 \mathrm{am}$ and awakening at $11 \mathrm{am}$. As with FASP, those with a strong DSP phenotype and family history have familial DSP (FDSP). Only one gene has been identified, $C R Y 1$, that confers an FDSP phenotype in an autosomal dominant 


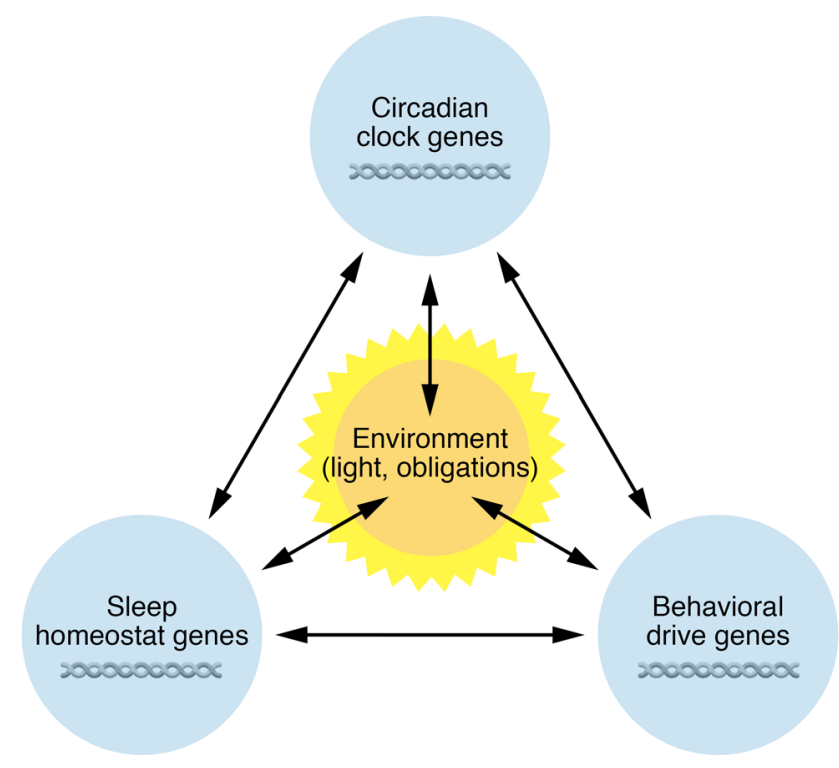

Figure 2. Contributors to timing and duration of sleep. Shown is a conceptual framework to demonstrate the multiple factors impacting the timing and duration of sleep. Genetic factors interact with environment, including light, social schedules, work and school obligations, and substances (alcohol, caffeine, medications). The result may be sleep duration and timing that differ from those suggested by genetics.

manner as a single gene mutation (58). Notably, if people with ASP or DSP are able to maintain their preferred schedule and do not have a complaint about it, the trait is not a disorder.

In addition to these monogenic phenotypes, genome-wide association studies using large data sets have identified a number of unvalidated variants associated with sleep length and timing. Jones et al., using large data sets from private sequencing companies, found significant genetic associations between certain loci and "morningness" (59). Some of these loci are in close proximity to known circadian genes, while others are only tangentially related to the circadian system, such as INADL, encoding a protein involved with photosensitive retinal cells (60). Understanding which genetic variants affect which particular genes will require biological studies to validate. Other studies have subsequently identified additional variants associated with chronotype (61). Though these variants individually have only a small influence on an individual's overall chronotype, their discovery reflects the system's complexity. For most individuals, the genetic contribution to chronotype is the cumulative effect of many genes that each make a small contribution (62).

Other inputs to the molecular clock may also affect chronotype or mask it, depending on environmental or biological context, further driving complexity (63). Indeed, genes associated with metabolic regulation and the immune system have also been implicated in circadian behaviors, indicating that research has only uncovered the tip of the iceberg $(16,61)$. Likewise, there is growing genetic evidence that clock-related genes - and, concomitantly, circadian regulation - affect other important biological processes in humans, including immune function and metabolism $(16,64$, 65). CLOCK and the PER proteins have been implicated in risk for addiction (66), BMAL1 is associated with diabetes (67), and PER3 variants have been linked to depression, among others $(56,68$,
69). The expanded availability of population-level sequencing data should continue to help researchers explore the diversity of human chronotypes and their relationship to overall human health.

It is important to note that genetics also influences sleep duration. Variation in this behavior can complicate identification of endogenous chronotype. Though not a circadian phenotype, sleep-related genes inherently affect an individual's sleep-wake timing, and therefore share some characteristics with circadian phenotypes. For example, a DEC2 mutation was the first to be identified in the familial natural short sleep (FNSS) phenotype (70), which manifests as an extremely early wake time in carriers, a behavior that is also apparent in extreme morning larks. However, these individuals stay up later than those with FASP and do not desire more sleep. Numerous other mutations have subsequently been described that lead to FNSS (71-73). The factors contributing to short duration of ad libitum sleep without detrimental effects, as is seen in FNSS, can be termed "behavioral drive." This drive, together with circadian clock genes, sleep homeostasis genes, and environmental factors, determines the timing and duration of sleep (Figure 2) - quantitative traits that are determined in part by the cumulative effects of numerous genetic variants. The degree to which each variant contributes likely varies considerably.

\section{Circadian rhythm sleep-wake disorders}

Circadian disorders arise when sleep-wake patterns, influenced by the circadian clock, are not in sync with desired sleep time, leading to a sleep or wake complaint. They include intrinsic circadian disorders, such as advanced sleep-wake phase disorder, delayed sleep-wake phase disorder, non-24 sleep-wake rhythm disorder, and irregular sleep-wake rhythm disorder; and extrinsic circadian disorders, such as shift work and jet lag disorder. Diagnosis is based on clinical history as well as sleep diary, actigraphy (a movement sensor that provides an objective estimate of sleep timing based on rest-activity rhythms), and dim-light melatonin onset (DLMO). Principles of treatment focus on resetting the circadian clock with zeitgebers, primarily light and melatonin, with timing based on the respective PRC. Under controlled laboratory conditions, 1 hour of bright light of approximately 8000 lux causes phase delay when delivered between about 3 hours before DLMO and about 9 hours after DLMO, and causes phase advance for the remaining portion of the curve $(41,74)$. This delay is maximal 1 to 3 hours after DLMO, leading to the recommendation of light at this time when attempting to delay. Advance is maximal approximately 10 hours after DLMO to 6 hours before, leading to the recommendation of morning light to achieve phase advance. Melatonin can also produce a phase response with a PRC about 12 hours out of sync with light and less potent than the PRC for light. This produces maximal delay around 2 hours before DLMO for melatonin doses of $0.5 \mathrm{mg}$ (75). Higher doses requiring a longer time for metabolism are thus given earlier. A $3 \mathrm{mg}$ dose has maximal phase-shifting impact approximately 5 hours before DLMO, and $10 \mathrm{mg}$ has lower phase-shifting ability, likely due to morning "spillover" causing competing impact on the PRC (76).

\section{Advanced sleep-wake phase disorder}

Those with ASP who have a sleep or wake complaint associated with the sleep pattern, such as early morning awakening, evening 
drowsiness, or insufficient sleep, have advanced sleep-wake phase disorder (ASWPD). For example, if someone with an extreme early chronotype tries to stay up late for social or family obligations but is not able to sleep correspondingly later given the strong biological drive to awaken early, the sleep duration is shortened (perhaps $11 \mathrm{pm}$ to $4: 30 \mathrm{am}$ ) and can result in excessive daytime sleepiness and other potential negative consequences of sleep curtailment. Diagnosis is based on clinical history with special attention paid to the patient's report of sleep timing during vacations or long weekends. Sleep diary and actigraphy are recommended for diagnosis. DLMO can also assist but is less clinically available and harder to perform. Prevalence of ASWPD in a sleep clinic population is estimated to be $0.04 \%$ (55). Treatment focuses on evening light such as sunlight or via a light box mimicking sunlight. However, the shifting predicted by the PRC contrived in experimental conditions may not directly translate into clinical practice, as data are limited and mixed regarding the benefit of evening light (77). Timed exercise, food, and melatonin may also help shift the clock, though there is limited experimental evidence to support their use in ASWPD. Improving timing of these non-photic zeitgebers may also help improve timing of light exposure. Given the paucity of good evidence and the difficulty of phase-shifting some individuals, accommodating the timing of social, familial, and work obligations to their rhythm may also be part of the treatment approach.

\section{Delayed sleep-wake phase disorder}

People with DSP and complaint regarding consequences of the trait, such as trouble falling asleep at the desired time and/or difficulty awakening for morning school or work obligations, have delayed sleep-wake phase disorder (DSWPD). DSWPD can be mistaken for sleep initiation insomnia, and differentiating these is essential to initiate optimal treatment. Thus, the clinical history should include sleep timing when an individual is allowed to sleep ad libitum. DSWPD is more common than ASWPD (78), most notably during adolescence, when sleep timing shifts later. Prevalence estimates for DSWPD range by definition and age but are $7 \%-16 \%$ in adolescents and young adults (79). Artificial light in the evening, lack of natural light, an intrinsic period longer than 24 hours, altered phase angle of entrainment, and possibly differential phototransduction to the SCN contribute to this higher prevalence of delayed sleep phase (80). Additional factors include school and work schedules that make sleeping later challenging.

Treatment for DSWPD is directed at modifying zeitgebers. Morning light (81-83) is routinely recommended, though optimal timing, dose, and duration are not yet understood. Afternoon or evening melatonin can also cause phase advance and is commonly combined with morning light in clinical practice despite limited data (77). Avoidance of evening light in the final 3 hours before sleep, when it is expected to lead to phase delay, may also be useful. Meals contribute to entrainment of peripheral oscillators and should be regulated as well $(84,85)$, as misalignment between the SCN and peripheral clocks in the gut may contribute to insulin resistance (86), though there are not yet sufficient data surrounding the impact of meal timing for standardized recommendations in treatment of DSWPD. As with ASWPD, and depending on the individual, these tools may not be adequate to fully shift to conventional sleep timing.

\section{Non-24-hour sleep-wake rhythm disorder}

Individuals with non-24-hour sleep-wake rhythm disorder (non-24; previously called free-running) have periods during which they are not able to stay on a 24-hour rhythm. Commonly these individuals drift progressively later. However, for those with a period of less than 24 hours, the drift can lead to progressively earlier sleep timing; this can manifest as daytime napping (87) and is typically mixed with periods of relative entrainment (88). Non-24 is most commonly diagnosed in blind individuals and occurs in the majority of those who lack light perception (89), as they do not have the input of light into the SCN, though it is possible to entrain to non-photic cues (90). Non-24 can occur in sighted individuals as well. Typically it occurs in those with DSWPD who shift into this less stable rhythm possibly as a result of weakened zeitgebers, and individuals can shift back and forth between DSWPD and non-24 (91). Symptoms of non-24 include alternating hypersomnia and insomnia with periods of relatively intact sleep. Non-24 is diagnosed with clinical history followed by sleep diary and actigraphy, demonstrating a progressively later or earlier sleep onset and awakening. DLMO at two time points several weeks apart can further support the diagnosis. Treatment focuses on timed melatonin administration. Melatonin must be initiated close to the onset of endogenous melatonin (92). A melatonin agonist, tasimelteon, has been approved for the treatment of non-24 in blind patients (93). Treatment for those who are sighted relies on timed sunlight or a light box, avoidance of evening light, and timed melatonin, though focus on weaker circadian cues such as meals, activity, and social interactions may help too. More research is needed into this condition.

\section{Irregular sleep-wake rhythm disorder}

Irregular sleep-wake rhythm disorder (ISWRD) is characterized by sleep spread out over the 24-hour day, during both day and night. This fractured sleep pattern causes insomnia at night and excessive sleepiness in the daytime. ISWRD typically occurs in individuals with developmental disorders, traumatic brain injury, or neurodegenerative disease. Reduced exposure to zeitgebers, reduced responsivity to circadian cues, and impaired SCN function are all thought to play a role in ISWRD pathophysiology (94). The diagnosis is confirmed with sleep diary and actigraphy that does not show a dominant sleep period (95). Treatment focuses on avoiding overnight light and improving exposure to bright light in the daytime to consolidate sleep overnight. Light therapy via a light box for at least 2 hours in the daytime has been shown to be beneficial (96). Melatonin administration likely benefits children with ISWRD, but its benefit in older adults with dementia is not clear $(94,97)$.

\section{Jet lag}

Jet lag is a temporary mismatch between local time and the internal clock resulting in sleep symptoms as well as malaise and gastrointestinal disturbance. When travel across at least two time zones results in insomnia, excessive daytime sleepiness, reduced total sleep time, and impaired daytime function or somatic symptoms 1 to 2 weeks after travel, this is characterized as jet lag disorder (98). Most individuals find westward travel easier than eastward travel (99). However, those with ASP typically find westward travel more difficult. Treatment focuses on timing of light exposure and melatonin administration. 


\section{Shift work disorder}

Many workers have a schedule that overlaps with their typical sleep time. If this results in insomnia, sleepiness, and reduced sleep duration, it is characterized as shift work disorder (SWD). People with SWD typically have sleep reduced by 1 to 4 hours owing to shift work and experience more health complaints than shift workers without SWD. There is great variation in ability to adapt to shift work, and there are not adequate data to predict who will experience SWD. Ten to thirty-eight percent of shift workers experience SWD (79). Symptoms typically resolve with resumption of a more traditional schedule, though chronic sleep trouble has been described.

\section{Burden of circadian misalignment}

The disorders described above lead to circadian misalignment, as does social jet lag, whereby an individual's social habits and circadian rhythms are misaligned, most often because of large differences in sleep timing on weekends and workdays (100). There is concern that all circadian disorders can lead to negative health outcomes, though this has been most clearly demonstrated with chronic shift work. Such disruptions can have far-reaching ramifications for nearly every tissue type of the body $(101,102)$. Both late chronotypes and shift workers have reported higher rates of psychiatric disorders such as depression (103, 104). Long-term, persistent shift work increases risk for dementia (105), type 2 diabetes $(36,106)$, cardiovascular disease (106), and cancer (107). Insulin sensitivity (106) and metabolic profiles (101,108-110) also appear to suffer. The development of these diseases can also exacerbate circadian disruption by compromising sleep and healthy routines, as is observed during neurodegenerative decline (52). These negative health outcomes have been found quite consistently in longitudinal studies, and the severity appears to correlate with the duration of shift work $(111,112)$.

Circadian disruption is a public health issue because, whether due to an intrinsic circadian disorder, shift work, jet lag, or other misalignment, it contributes to the development of adverse effects on health, including (but not limited to) heart disease $(106,113)$, neurodegeneration (114), cancer (107), and mental health (52,101, $105,107,115-117)$. In addition to these effects on the individuals themselves, circadian rhythm disruption can lead to adverse outcomes for society: nurses who regularly work long or erratic shifts display poorer performance (112), and car accidents increase in likelihood corresponding with duration of chronic sleep restriction $(118,119)$. A recent long-term longitudinal study found that people with evening chronotypes who operated on a morning work schedule were more likely to require disability assistance (120). It is still unknown to what degree the health consequences described above are attributable to the disrupted sleep concomitant with shift work rather than circadian desynchronization itself. But evening chronotype appears to be correlated with multiple negative health outcomes, possibly owing to a social/biological clock misalignment or substantial social jet lag $(109,121)$.

\section{Next steps}

Measures aimed at improving social flexibility for a diversity of chronotypes or wiser shift scheduling may work to improve circadian misalignment (122-124). To start, the evidence suggests that we should make efforts to reduce social stigmatization of late chronotypes, as traditionally those who habitually sleep late into the day may be viewed as merely lazy. An important example population to which this suggestion could immediately be applied is adolescents. Adolescents typically have a later chronotype than adults $(49,125)$, and studies have indicated that cognitive performance - and, correspondingly, overall education - improves in adolescents allowed a later school start time (126).

\section{Conclusion}

Genetics drive human circadian phase and period length and guides the rhythmicity of numerous circadian physiological processes. Genetic variations, along with environmental influences, result in varying chronotypes and phenotypic extremes. Some affected people adapt to and function well with extreme chronotypes and lead healthy lives, while others may find the same chronotype leads to troublesome circadian disorders, desynchronization, social jet lag, and sleep deficits. Any quantitative definitional cutoff for the timing of a circadian "disorder" (e.g., biological wake time of 0400 hours) is therefore arbitrary and dependent on the individual's context. These extremes of chronotype need not be viewed inherently as disorders, but can be seen as a natural behavioral trait to be managed on a case-by-case basis through interventions such as timed exposure to sunlight in order to shift phase. What is clear, however, is that desynchrony due to differences between endogenous clock timing and lifestyle and societal factors - as can exist in people with extreme chronotypes, shift workers, and adolescents - leads to sleep deprivation and associated adverse health outcomes. Framing some degree of circadian variation as natural and not merely due to preference should allow and encourage social interventions that improve the health of all individuals, regardless of morningness or eveningness.

\section{Acknowledgments}

This work was supported by NIH grants NS099333, NS072360, and NS104782 to LJP and YHF, and by the William Bowes Neurogenetics Fund to LJP and YHF.

Address correspondence to: Ying-Hui Fu or Louis J. Ptáček, Department of Neurology, University of California, San Francisco, 548B Rock Hall MC-2922 (YHF) or 548F Rock Hall MC-2922 (LJP), 1550 4th Street, San Francisco, California 94143, USA. Email: Ying-Hui.Fu@ucsf.edu (YHF); Email: ljp@ucsf.edu (LJP).

\footnotetext{
1. Konopka RJ, Benzer S. Clock mutants of Drosophila melanogaster. Proc Natl Acad Sci US A. 1971;68(9):2112-2116.

2. Johnson $\mathrm{CH}$, et al. Structural insights into a circadian oscillator. Science. 2008;322(5902):697-701.

3. Bargiello TA, et al. Restoration of circadian
}

behavioural rhythms by gene transfer in Drosophila. Nature. 1984;312(5996):752-754.

4. Siwicki KK, et al. Antibodies to the period gene product of Drosophila reveal diverse tissue distribution and rhythmic changes in the visua system. Neuron. 1988;1(2):141-150.
5. Czeisler CA, Gooley JJ. Sleep and circadian rhythms in humans. Cold Spring Harb Symp Quant Biol. 2007;72:579-597.

6. Pandi-Perumal SR, et al. Dim light melatonin onset (DLMO): a tool for the analysis of circadian phase in human sleep and chronobiological dis- 
orders. Prog Neuropsychopharmacol Biol Psychiatry. 2007;31(1):1-11.

7. Lewis $\mathrm{P}$, et al. Food as a circadian time cue - evidence from human studies. Nat Rev Endocrinol. 2020;16(4):213-223.

8. Lewis P, et al. Exercise time cues (zeitgebers) for human circadian systems can foster health and improve performance: a systematic review. $B M J$ Open Sport Exerc Med.2018;4(1):e000443.

9. Czeisler CA, et al. Bright light resets the human circadian pacemaker independent of the timing of the sleep-wake cycle. Science. 1986;233(4764):667-671.

10. Czeisler CA, et al. Stability, precision, and near24-hour period of the human circadian pacemaker. Science. 1999;284(5423):2177-2181.

11. Saper CB. The central circadian timing system. Curr Opin Neurobiol. 2013;23(5):747-51.

12. Golombek DA, et al. The times they're a-changing: effects of circadian desynchronization on physiology and disease. J Physiol Paris. 2013;107(4):310-322.

13. Uchiyama M, Lockley SW. Non-24-hour sleepwake rhythm disorder in sighted and blind patients. Sleep Med Clin. 2015;10(4):495-516.

14. Hozer C, et al. Survival is reduced when endogenous period deviates from $24 \mathrm{~h}$ in a non-human primate, supporting the circadian resonance theory. Sci Rep. 2020;10(1):18002.

15. Pittendrigh CS, Daan S. A functional analysis of circadian pacemakers in nocturnal rodents. J Comp Physiol. 1976;106(3):223-252.

16. Zhang L, et al. Diversity of human clock genotypes and consequences. Prog Mol Biol Transl Sci. 2013;119:51-81.

17. Roenneberg T, et al. Life between clocks: daily temporal patterns of human chronotypes. J Biol Rhythms. 2003;18(1):80-90.

18. Hofstra WA, de Weerd AW. How to assess circadian rhythm in humans: a review of literature. Epilepsy Behav. 2008;13(3):438-444.

19. Duffy JF, et al. Sex difference in the near-24-hour intrinsic period of the human circadian timing system. Proc Natl Acad Sci U S A. 2011;108(suppl 3):15602-15608.

20. Phillips AJK, et al. High sensitivity and interindividual variability in the response of the human circadian system to evening light. Proc Natl Acad Sci U S A. 2019;116(24):12019-12024.

21. Fischer D, et al. Chronotypes in the US - influence of age and sex. PLoS One. 2017;12(6):e0178782.

22. Wright KP, et al. Entrainment of the human circadian clock to the natural light-dark cycle. Curr Biol. 2013;23(16):1554-1558.

23. Stothard ER, et al. Circadian entrainment to the natural light-dark cycle across seasons and the weekend. Curr Biol. 2017;27(4):508-513.

24. Zavada A, et al. Comparison of the Munich Chronotype Questionnaire with the Horne-Östberg's Morningness-Eveningness score. Chronobiol Int. 2005;22(2):267-278.

25. Toh KL, et al. An hPer2 phosphorylation site mutation in familial advanced sleep phase syndrome. Science. 2001;291(5506):1040-1043.

26. Jones CR, et al. Familial advanced sleep-phase syndrome: a short-period circadian rhythm variant in humans. Nat Med.1999;5(9):1062-1065.

27. Xu Y, et al. Functional consequences of a CKI $\delta$ mutation causing familial advanced sleep phase syndrome. Nature. 2005;434(7033):640-644.

28. Lowrey PL, Takahashi JS. Genetics of circadian rhythms in mammalian model organisms. $A d v$ Genet. 2011;74:175-230.

29. Lowrey PL, Takahashi JS. Mammalian circadian biology: elucidating genome-wide levels of temporal organization. Annu Rev Genomics Hum Genet. 2004;5:407-441.

30. Solt LA, et al. The REV-ERBs and RORs: molecular links between circadian rhythms and lipid homeostasis. Future Med Chem. 2011;3(5):623-638.

31. Padmanabhan $\mathrm{K}$, et al. Feedback regulation of transcriptional termination by the mammalian circadian clock PERIOD complex. Science. 2012;337(6094):599-602.

32. Koike $\mathrm{N}$, et al. Transcriptional architecture and chromatin landscape of the core circadian clock in mammals. Science. 2012;338(6105):349-354.

33. Zhang R, et al. A circadian gene expression atlas in mammals: implications for biology and medicine. Proc Natl Acad Sci U S A. 2014;111(45):16219-16224.

34. Eng GWL, et al. Site-specific phosphorylation of casein kinase $1 \delta$ (CK1ס) regulates its activity towards the circadian regulator PER2. PLoS One. 2017;12(5):e0177834.

35. Bass J, Takahashi JS. Circadian integration of metabolism and energetics. Science. 2010;330(6009):1349-1354.

36. Ikegami K, et al. Interconnection between circadian clocks and thyroid function. Nat Rev Endocrinol. 2019;15(10):590-600.

37. Ruby NF, et al. Scheduled feeding restores memory and modulates c-Fos expression in the suprachiasmatic nucleus and septohippocampal complex. Sci Rep. 2017;7(1):6755.

38. Ferrell JM, Chiang JYL. Circadian rhythms in liver metabolism and disease. Acta Pharm Sin B. 2015;5(2):113-122.

39. Eckel-Mahan K, Sassone-Corsi P. Metabolism and the circadian clock converge. Physiol Rev. 2013;93(1):107-135.

40. Minors DS, et al. A human phase-response curve to light. Neurosci Lett. 1991;133(1):36-40.

41. St Hilaire MA, et al. Human phase response curve to a $1 \mathrm{~h}$ pulse of bright white light. J Physiol. 2012;590(13):3035-3045.

42. Chellappa SL, et al. Can light make us bright? Effects of light on cognition and sleep. Prog Brain Res. 2011;190:119-33.

43. Tähkämö L, et al. Systematic review of light exposure impact on human circadian rhythm. Chronobiol Int. 2019;36(2):151-170.

44. Cho Y, et al. Effects of artificial light at night on human health: a literature review of observational and experimental studies applied to exposure assessment. Chronobiol Int. 2015;32(9):1294-1310.

45. Roenneberg T, et al. Epidemiology of the human circadian clock. Sleep Med Rev. 2007;11(6):429-438.

46. Roenneberg T, et al. A marker for the end of adolescence. Curr Biol. 2004;14(24):R1038-R1039.

47. Nakamura TJ, et al. Age-related changes in the circadian system unmasked by constant conditions. eNeuro. 2015;2(4):ENEURO.0064-15.2015.

48. Chen Z, et al. Small molecule modifiers of circadian clocks. Cell Mol Life Sci. 2013;70(16):2985-2998.
49. Duffy JF, et al. Aging and circadian rhythms. Sleep Med Clin. 2015;10(4):423-434.

50. Leng $\mathrm{Y}$, et al. Association between circadian rhythms and neurodegenerative diseases. Lancet Neurol. 2019;18(3):307-318.

51. Leng $Y$, et al. Association of circadian abnormalities in older adults with an increased risk of developing Parkinson disease. JAMA Neurol. 2020;77(10):1270-1278.

52. Musiek ES. Circadian clock disruption in neurodegenerative diseases: cause and effect? Front Pharmacol. 2015;6:29.

53. Musiek ES, et al. Sleep, circadian rhythms, and the pathogenesis of Alzheimer disease. Exp Mol Med. 2015;47(3):e148.

54. Chang HC, et al. SIRT1 mediates central circadian control in the SCN by a mechanism that decays with aging. Cell. 2013;153(7):1448-1460.

55. Curtis BJ, et al. Extreme morning chronotypes are often familial and not exceedingly rare: the estimated prevalence of advanced sleep phase, familial advanced sleep phase, and advanced sleep-wake phase disorder in a sleep clinic population. Sleep. 2019;42(10):zsz148.

56. Zhang L, et al. A PERIOD3 variant causes a circadian phenotype and is associated with a seasonal mood trait. Proc Natl Acad Sci U S A. 2016;113(11):E1536-E1544.

57. Kurien P, et al. TIMELESS mutation alters phase responsiveness and causes advanced sleep phase. Proc Natl Acad Sci U S A. 2019;116(24):12045-12053.

58. Patke A, et al. Mutation of the human circadian clock gene CRY1 in familial delayed sleep phase disorder. Cell. 2017;169(2):203-215.

59. Jones SE, et al. Genome-wide association analyses in 128,266 individuals identifies new morningness and sleep duration loci. PLoS Genet. 2016;12(8):e1006125.

60. Mazzotta G, et al. Fly cryptochrome and the visual system. Proc Natl Acad Sci U S A. 2013;110(15):6163-6168.

61. Kalmbach DA, et al. Genetic basis of chronotype in humans: insights from three landmark GWAS. Sleep. 2017;40(2):zsw048.

62. Shimomura K, et al. Genome-wide epistatic interaction analysis reveals complex genetic determinants of circadian behavior in mice. Genome Res. 2001;11(6):959-980.

63. Azzi A, et al. Circadian behavior is light-reprogrammed by plastic DNA methylation. Nat Neurosci. 2014;17(3):377-382.

64. Patel SA, et al. Calorie restriction regulates circadian clock gene expression through BMAL1 dependent and independent mechanisms. Sci Rep. 2016;6(1):25970.

65. Nguyen KD, et al. Circadian gene Bmal1 regulates diurnal oscillations of Ly6Chi inflammatory monocytes. Science. 2013;341(6153):1483-1488.

66. Falcón E, McClung CA. A role for the circadian genes in drug addiction. Neuropharmacology. 2009;56(suppl 1):91-96.

67. Woon PY, et al. Aryl hydrocarbon receptor nuclear translocator-like (BMAL1) is associated with susceptibility to hypertension and type 2 diabetes. Proc Natl Acad Sci U S A. 2007;104(36):14412-14417.

68. Shi SQ, et al. Molecular analyses of circadi- 
an gene variants reveal sex-dependent links between depression and clocks. Transl Psychiatry. 2016;6(3):e748.

69. Weiss C, et al. Sleep quality, sleep structure, and PER3 genotype mediate chronotype effects on depressive symptoms in young adults. Front Psychol. 2020;11:2028.

70. He Y, et al. The transcriptional repressor DEC2 regulates sleep length in mammals. Science. 2009;325(5942):866-870.

71. Shi $\mathrm{G}$, et al. A rare mutation of $\beta_{1}$-adrenergic receptor affects sleep/wake behaviors. Neuron. 2019;103(6):1044-1055.

72. Xing L, et al. Mutant neuropeptide S receptor reduces sleep duration with preserved memory consolidation. Sci Transl Med. 2019;11(514):2014.

73. Shi G, et al. Mutations in metabotropic glutamate receptor 1 contribute to natural short sleep trait. Curr Biol. 2021;31(1):13-24.

74. Khalsa SBS, et al. A phase response curve to single bright light pulses in human subjects. J Physiol. 2003;549(3):945-952.

75. Lewy AJ, et al. Melatonin shifts human circadian rhythms according to a phase-response curve. Chronobiol Int. 1992;9(5):380-392.

76. Burgess HJ, et al. A three pulse phase response curve to three milligrams of melatonin in humans. J Physiol. 2008;586(2):639-647.

77. Auger RR, et al. Clinical practice guideline for the treatment of intrinsic circadian rhythm sleepwake disorders: advanced sleep-wake phase disorder (ASWPD), delayed sleep-wake phase disorder (DSWPD), non-24-hour sleep-wake rhythm disorder (N24SWD), and irregular sleep-wake rhythm disorder (ISWRD). An update for 2015: an American Academy of Sleep Medicine clinical practice guideline. JClin Sleep Med. 2015;11(10):1199-1236.

78. Paine SJ, et al. Identifying advanced and delayed sleep phase disorders in the general population: a national survey of New Zealand adults. Chronobiol Int . 2014;31(5):627-636.

79. International Classification of Sleep Disorders. 2nd ed. American Academy of Sleep Medicine; 2005.

80. Abbott SM, et al. Melanopsin-dependent phototransduction is impaired in delayed sleep-wake phase disorder and sighted non-24-hour sleepwake rhythm disorder. Sleep. 2021;44(2):zsaa184.

81. Rosenthal NE, et al. Phase-shifting effects of bright morning light as treatment for delayed sleep phase syndrome. Sleep. 1990;13(4):354-361.

82. Richardson C, et al. A randomised controlled tria of bright light therapy and morning activity for adolescents and young adults with delayed sleepwake phase disorder. Sleep Med. 2018;45:114-123.

83. Cole RJ, et al. Bright-light mask treatment of delayed sleep phase syndrome. J Biol Rhythms. 2002;17(1):89-101.

84. Stokkan KA, et al. Entrainment of the circadian clock in the liver by feeding. Science. 2001;291(5503):490-493.

85. Wehrens SMT, et al. Meal timing regulates the human circadian system. Curr Biol. 2017;27(12):1768-1775.

86. Stenvers DJ, et al. Circadian clocks and insulin resistance. Nat Rev Endocrinol. 2019;15(2):75-89.

87. Lockley SW, et al. Sleep and activity rhythms are related to circadian phase in the blind. Sleep. 1999;22(5):616-623.
88. Emens JS, et al. Non-24-hour disorder in blind individuals revisited: Variability and the influence of environmental time cues. Sleep. 2013;36(7):1091-1100.

89. Flynn-Evans EE, et al. Circadian rhythm disorders and melatonin production in 127 blind women with and without light perception. J Biol Rhythms. 2014;29(3):215-224.

90. Klerman EB, et al. Nonphotic entrainment of the human circadian pacemaker. Am J Physiol Regul Integr Comp Physiol. 1998;274(4):R991-R996.

91. Abbott SM. Non-24-hour sleep-wake rhythm disorder. Neurol Clin. 2019;37(3):545-552.

92. Lockley SW, et al. Melatonin administration can entrain the free-running circadian system of blind subjects. JEndocrinol. 2000;164(1):R1-R6.

93. Lockley SW, et al. Tasimelteon for non-24-hour sleep-wake disorder in totally blind people (SET and RESET): two multicentre, randomised, double-masked, placebo-controlled phase 3 trials. Lancet . 2015;386(10005):1754-1764.

94. Zee PC, Vitiello MV. Circadian rhythm sleep disorder: irregular sleep wake rhythm. Sleep Med Clin. 2009;4(2):213-218.

95. International Classification of Sleep Disorders. 3rd ed. American Academy of Sleep Medicine; 2014.

96. Mishima K, et al. Morning bright light therapy for sleep and behavior disorders in elderly patients with dementia. Acta Psychiatr Scand. 1994;89(1):1-7.

97. Singer C, et al. A multicenter, placebo-controlled trial of melatonin for sleep disturbance in Alzheimer's disease. Sleep. 2003;26(7):893-901.

98. Sateia MJ. International classification of sleep disorders-third edition highlights and modifications. Chest. 2014;146(5):1387-1394.

99. Drake CL, Wright KP. Shift work, shift-work disorder, and jet lag. In: Kryfer M, et al., eds. Principles and Practice of Sleep Medicine. 5th ed. Elsevie Inc.; 2010:784-798.

100.Wittmann M, et al. Social jetlag: misalignment of biological and social time. Chronobiol Int. 2006;23(1-2):497-509.

101.Parsons MJ, et al. Social jetlag, obesity and metabolic disorder: investigation in a cohort study. Int JObes. 2015;39(5):842-848.

102. Rivera AS, et al. Shift work and long work hours and their association with chronic health conditions: a systematic review of systematic reviews with meta-analyses. PLoS One. 2020;15(4):e0231037.

103. Alvaro PK, et al. The independent relationships between insomnia, depression, subtypes of anxiety, and chronotype during adolescence. Sleep Med. 2014;15(8):934-941.

104.Germain A, Kupfer DJ. Circadian rhythm disturbances in depression. Hum Psychopharmacol Clin Exp. 2008;23(7):571-585.

105. Jørgensen JT, et al. Shift work and incidence of dementia: a Danish Nurse Cohort study. Alzheimers Dement. 2020;16(9):1268-1279.

106. Kervezee L, et al. Metabolic and cardiovascular consequences of shift work: the role of circadian disruption and sleep disturbances. Eur J Neurosci. 2020;51(1):396-412.

107. Hansen J, Stevens RG. Case-control study of shift-work and breast cancer risk in Danish nurses: impact of shift systems. Eur JCancer.
2012;48(11):1722-1729.

108. Taheri S, et al. Short sleep duration is associated with reduced leptin, elevated ghrelin, and increased body mass index. PLoS Med. 2004;1(3):e62.

109. Partonen T. Chronotype and health outcomes Curr Sleep Med Reports. 2015;1(4):205-211.

110.Eckel-Mahan K, Sassone-Corsi P. Metabolism control by the circadian clock and vice versa. $\mathrm{Nat}$ Struct Mol Biol. 2009;16(5):462-467.

111. Haus E, Smolensky M. Biological clocks and shift work: circadian dysregulation and potential long-term effects. Cancer Causes Control. 2006;17(4):489-500.

112. Fitzpatrick JM, et al. Shift work and its impact upon nurse performance: current knowledge and research issues. J Adv Nurs. 1999;29(1):18-27.

113. Kervezee L, et al. Simulated night shift work induces circadian misalignment of the human peripheral blood mononuclear cell transcriptome. Proc Natl Acad Sci U S A. 2018;115(21):5540-5545.

114. Mander BA, et al. Sleep and human aging. Neuron. 2017;94(1):19-36.

115. Gangwisch JE. Epidemiological evidence for the links between sleep, circadian rhythms and metabolism. Obes Rev. 2009;10(suppl 2):37-45.

116. Levandovski R, et al. Depression scores associate with chronotype and social jetlag in a rural population. Chronobiol Int. 2011;28(9):771-778.

117. Mansour HA, et al. Circadian genes and bipolar disorder. Ann Med. 2005;37(3):196-205

118. Van Dongen HPA, et al. The cumulative cost of additional wakefulness: dose-response effects on neurobehavioral functions and sleep physiology from chronic sleep restriction and total sleep deprivation. Sleep. 2003;26(2):117-126.

119. McCoy JG, Strecker RE. The cognitive cost of sleep lost. Neurobiol Learn Mem. 2011;96(4):564-582.

120. Räihä T, et al. Evening chronotype is associated with poor work ability and disability pensions at midlife: a Northern Finland Birth Cohort 1966 Study [published online February 23, 2021] Occup Environ Med. https://doi.org/10.1136/ oemed-2020-107193.

121. Didikoglu A, et al. Longitudinal change of sleep timing: association between chronotype and longevity in older adults. Chronobiol Int. 2019;36(9):1285-1300.

122. Lowden A, et al. Working time society consensus statements: evidence based interventions using light to improve circadian adaptation to working hours. Ind Health. 2019;57(2):213-227.

123. Smith MR, et al. Practical interventions to promote circadian adaptation to permanent night shift work: study 4. J Biol Rhythms. 2009;24(2):161-172.

124.Van Reeth O. Sleep and circadian disturbances in shift work: strategies for their management. Horm Res. 1998;49(3-4):158-162.

125. Laberge L, et al. Sleep and circadian phase characteristics of adolescent and young adult males in a naturalistic summertime. Chronobiol Int. 2000;17(4):489-501.

126. Lo JC, et al. Sustained benefits of delaying school start time on adolescent sleep and well-being. Sleep. 2018;41(6):zsy052.

127. Ashbrook LH, et al. Genetics of the human circadian clock and sleep homeostat. Neuropsychopharmacology. 2020;45(1):45-54 